\title{
In Situ Mechanical Loading and Neutron Bragg-edge Imaging, Applied to Polygranular Graphite On IMAT@ISIS
}

\author{
T. A. C. Zillhardt ${ }^{1}$ (D) G. Burca² $\cdot$ D. Liü ${ }^{3} \cdot$ T. J. Marrow ${ }^{1}$
}

Received: 27 April 2021 / Accepted: 8 July 2021 / Published online: 9 August 2021

(c) The Author(s) 2021

\begin{abstract}
Background Bragg edge imaging have seen significant developments in the last decade with the availability of new timeresolved detectors, however, there have been no studies of changes in local coherent scattering from grain reorientation and deformation with load. Such damage accommodation mechanism may occur in (quasi)-brittle materials.

Objective We developed a novel method using in-situ Bragg imaging at the ISIS spallation neutron and muon source on the IMAT (Imaging and MATerials science and engineering) instrument using an energy-resolved detector setup. We collected and analysed data of a proof-of-concept experiment demonstrating the use of the method.

Methods We have developed a loading apparatus that addresses the constraints posed by Bragg imaging, allowing us to resolve features in the material microstructure. We use energy-resolved neutron imaging to obtain images in energy bins and we have developed a set of codes to register and correlate these images, as well as detect changes in local coherent scattering, in situ.

Results Preliminary results from this method on Gilsocarbon nuclear graphite allow qualitative observation of local changes in Bragg contrast, which may be due to deformation or grain reorientation.

Conclusions We have demonstrated that we can track changes in local coherent scattering under mechanical load, with sufficient resolution to track features with a size above 100 microns. This method, apparatus and accompanying codes may be used on the IMAT instruments by users interested to better understand deformation in their materials.
\end{abstract}

Keywords Neutron diffraction · Bragg imaging · Digital Image Correlation · Graphite · Crystal deformation

\section{Introduction}

Synchrotron X-ray and neutron sources offer unique and complementary capabilities to examine the deformation of engineering materials where information is required at multiple length scales, from the bulk to the crystalline structure. In particular, spallation neutron sources with time-of-flight capabilities allow the acquisition of energy-resolved datasets, and beamlines have been commissioned specifically to address engineering problems using these advantages, such

T. A. C. Zillhardt

thomaszillhardt@cantab.net

1 Department of Materials, University of Oxford, Parks Road, Oxford OX13PH, UK

2 Rutherford Appleton Laboratory, STFC, ISIS Facility, Harwell, UK

3 HH Wills Physics Laboratory Tyndall Avenue, Bristol BS8 1TL, UK as the IMAT (Imaging and MATerials science and engineering) instrument [1] on the ISIS ${ }^{1}$ neutron and muon source, at the Rutherford Appleton Laboratory of the Science and Technology Facilities Council (STFC) on the Harwell Science and Innovation Campus in Oxfordshire [2]. Polygranular graphite has been used in critical structural components in operating nuclear fission reactors worldwide and is proposed for use in future advanced reactors. The deformation and failure mechanisms of graphite, both before and after irradiation, are of great importance to the understanding of in-service behaviour and the assurance of safety [3-5]. It has therefore been selected as the material for this proofof-concept study that aims to observe the effects of grain deformation and re-orientation on local changes in coherent scattering.

IMAT is a recently commissioned imaging beamline that offers a time-integrated neutron flux of $4 \times 10^{7} \mathrm{n} / \mathrm{cm}^{2} / \mathrm{s}$ with a $40 \mathrm{~mm}$ pinhole aperture thanks to a straight neutron guide

\footnotetext{
1 ISIS is the name of the facility and is not an acronym.
} 
Fig. 1 Beam spectra from IMAT Monitor 5, located $49.5 \mathrm{~m}$ from the moderator (run number 15209: Scan 5, Load $4 \mathrm{kN}, 120 \mu \mathrm{A}$ )

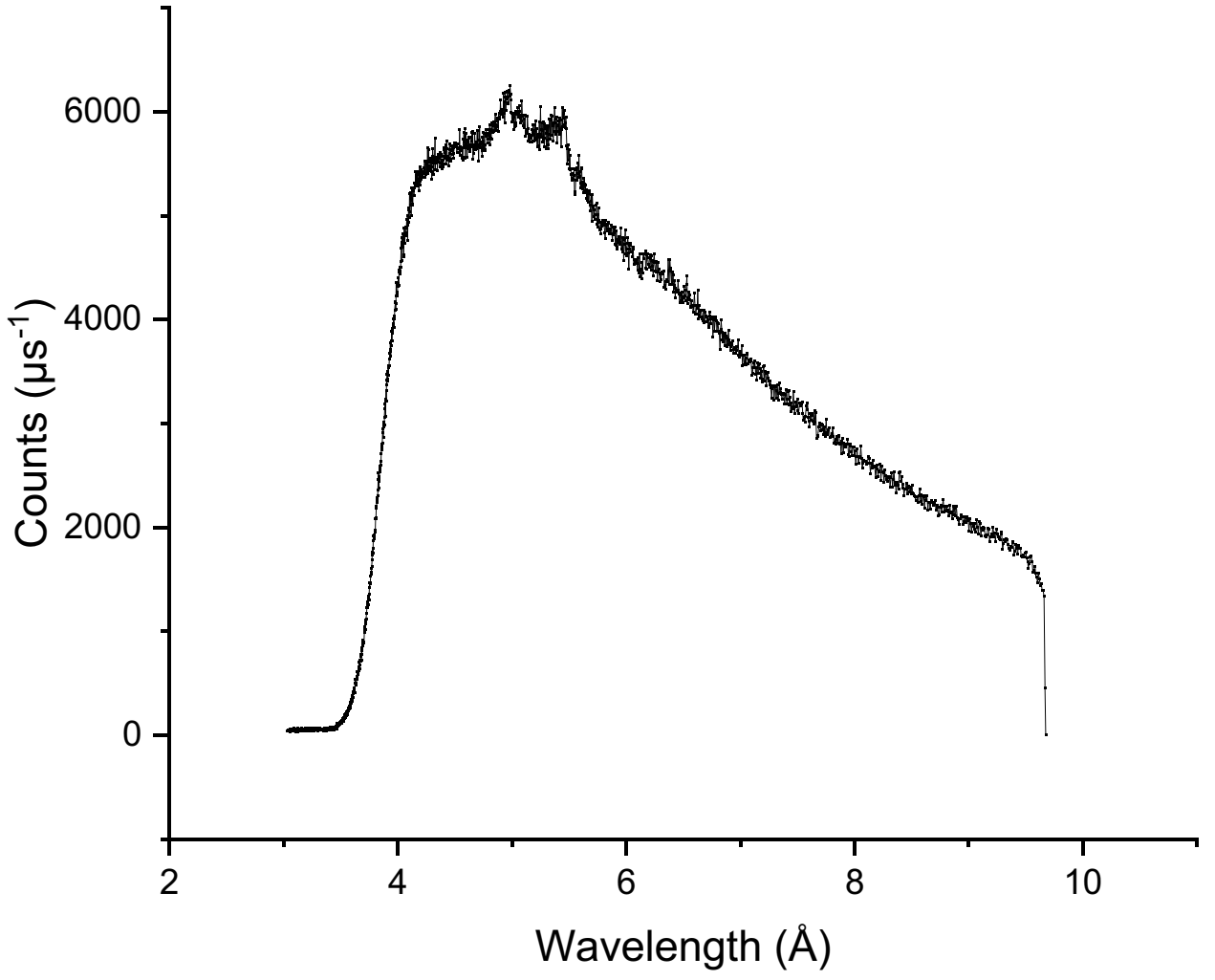

$[1,6]$. Between the source and the sample stage position, distanced by $56 \mathrm{~m}$, a $20 \mathrm{~Hz}$ chopper filters the fast neutrons and gamma radiation at $12.75 \mathrm{~m}$ from the moderator while two $10 \mathrm{~Hz}$ double-disc choppers at respectively 12.2 and $20.4 \mathrm{~m}$ from the moderator are used to select the wavelength bands and avoid frame-overlap. They allow users to define and shift the pulse spectrum as required to suit the material being studied. Error! Reference source not found. The neutron guide ends just before the experimental hutch with a pinhole selector offering apertures of 20, 30, 40, 60 and $80 \mathrm{~mm}$, which provides a quasi-parallel beam with a $L_{P} / D$ specific divergence, where $D$ is the aperture and $L_{P}$ the length to the neutron camera from the pinhole. The resolution, $r$, is independent of the camera pixel size and can be calculated with equation (1), where $l$ is the distance from the active area of the detector to the centreline of the sample.

$r=\frac{l}{L_{P} / D}$

Within the hutch, an in-vacuum tube carries the neutrons to the sample area and comprises a set of 5 beam-limiting boron carbide jaws. The last retractable neutron monitor on the beam, Monitor 5, is located just before the end of the tube, and allows one to check the shape of the flux. Figure 1 is an example of the neutron count from Monitor 5 on IMAT. Although it is possible to use a white beam and shift the pulse with appropriate chopper parameters, this has limitations that need to be accounted for when studying materials with energies away from the peak flux. This is due to the need to avoid neutron pulse overlap and the maximum wavelength band, $\Delta \lambda$, is set by equation (2) [6].

$\Delta \lambda=\frac{3957}{L_{S} f}$

where $f$ is the repetition rate and $L_{S}$ is the distance from the source to the detector.

Bragg-edge imaging is a fast-developing acquisition tool that provides energy-resolved information of internal structures in engineering components. Time-of-flight energyresolved neutron imaging using different sensors has been initially used for in-situ strain analysis and 2D single crystal Bragg-dip mapping [7-10]. More recent developments of insitu wavelength-dependent neutron imaging have been able to accurately determine the lattice spacing, lattice strains and coefficient of thermal expansion of metallic powder samples [11]. This has been made possible by the development of Microchannel Plate (MCP) detectors capable of time resolutions below $10 \mathrm{~ns}$. The MCP detector on IMAT, developed at the University of California at Berkley [12], is composed of a tile of four Timepix ASIC sensor chips offering a field of view of $28 \times 28 \mathrm{~mm}$ with a pixel size of $55 \mu \mathrm{m}$ at event rates up to $200 \mathrm{MHz}$.

A relatively unexplored use of Bragg-edge imaging is direct image processing and analysis of extinction spots for 
crystals in the Bragg-condition. This use of energy-resolved neutron radiographs is particularly sensitive to the resolution defined by equation (1) and is most suitable for materials with grains sizes or domains over $100 \mu \mathrm{m}$ in size. Several methods can be used to investigate grain orientations. Electron Back-Scattered Diffraction (EBSD) has a number of limitations including sample size and the requirement to polish the examined surface, and it is not very suitable for bulk samples of materials such as graphite [13]. For the characterisation of grains within the microstructure, Three-Dimensional X-Ray Diffraction (3DXRD) grain reconstruction, as well as Diffraction Contrast Tomography (DCT), have proven effective for metals and materials with a low mosaic spread [14-17]. DCT can be applied with in-situ loading or heating and has the potential to provide information on the grain strain state [18]. The DCT technique has been generalized to neutron imaging with the development of the ToF-3DND (Time of Flight Three Dimensional Neutron Diffraction) grain mapping method [19].

In this work, we propose a novel method that is suited to investigate the effects of grain reorientation and deformation in polycrystalline samples with coarse grain size or grain domains (from $\sim 100 \mu \mathrm{m}$ ) using in situ time-of-flight energy-resolved neutron imaging. A novel mechanical loading device for high resolution imaging has been designed, and tools for advanced image analysis have been developed, to detect the response to applied mechanical stress of microstructural features that are close to the Bragg condition. Results are presented from the proof-of-concept experiment $\mathrm{n}^{\circ} \mathrm{RB} 1910517$ [20] on the IMAT instrument at the STFCISIS neutron and spallation source.

As the beam passes through the sample and neutrons are collected on the MCP detector, all those neutrons that are diffracted via coherent scattering in the crystal (i.e., at wavelengths depending on specific crystal planes and their orientations) will not be recorded. Specifically, when the neutron wavelength is much larger than twice the interplanar spacing of the periodic $\{\mathrm{hkl}\}$ planes (i.e., $d_{h k l}$ ), there is no coherent scattering, and the transmission varies with the square of the neutron wavelength. At wavelengths where the Bragg condition is just satisfied by suitably oriented planes there is a marked change in the transmitted intensity (i.e., a Bragg edge) [21]. On a radiograph at a specific energy the reduced intensity from grains in which the neutrons that have been scattered will form extinction spots. The sensitivity of the intensity of these spots to changes in crystal orientation, or changes in scattering due to the disruption of coherency by plastic deformation [22], will be strongest for energies close to the Bragg edge. We aim to map and observe the changes in these features as a mechanical load is applied.

Gilsocarbon graphite was examined as there is interest in understanding its deformation mechanisms under mechanical load. Graphite is a polygranular material used in nuclear fission reactors as a neutron moderator and as a reflector, such as the fleet of UK Advanced Gas Cooled Reactors (AGR) [4] and planned 4th Gen Very High Temperature and Molten Salt Reactors (VHTR/MSR) [5]. These graphites have an important role in the core structural integrity and the lifespan of these nuclear reactors. Gilsocarbon is made by impregnation of calcined Gilsonite particles with a pitch binder [23, 24], resulting in a coarse microstructure with domains of aligned crystallites [25]. Graphite has a high neutron elastic scattering cross-section [26], a strong Bragg edge associated with its basal plane [27], making it an ideal candidate for this study. There is a lack of evidence for the mechanisms of deformation of bulk polygranular graphites such as Gilsocarbon, which exhibits a change in deformation behaviour at elevated temperatures, leading to improved toughness and tensile strength [28]. Due to its polycrystalline nature and the $\mathrm{ABAB}$ stacking in hexagonal graphite crystals, shear by basal dislocation glide occurs readily [29] and graphite crystals also exhibit kink band formation [30]. Twinning in graphite, first reported in [31] and observed by optical microscopy [32], is theorised as a rotation of approximately $20^{\circ}$ about $<1 \overline{1} 01>$ [33]. Twinning under load in natural graphite flakes has been demonstrated with electron microscopy [34] and the analysis of ion-beam irradiation of pyrolytic graphite has demonstrated the formation of a novel reoriented crystal vein structure due to basal dislocations [35]. It has also been reported, for neutron-irradiated synthetic graphite, that twins recover once the load is removed [36] so twinning may be an elastic deformation mechanism. Observations of the local changes in coherent scattering may provide a way to obtain quantitative data on the mechanisms of grain reorientation by twinning, and the role of plastic deformation. This would provide essential information for the microstructural models that are needed to understand the effects of irradiation and temperature on graphite properties in order to support the design and operation of advanced fission reactors.

\section{Experiment}

\section{Design of the Loading Rig}

The method consists of a neutron energy-resolved acquisition of a specimen while applying mechanical compression. We apply a small preload to the sample and subsequently proceed to radiography acquisition. This is followed by a series of loading stages, each associated with radiograph acquisition of equal duration. The radiograph image resolution is independent of the detector pixel size and varies principally with the sample to detector active area distance, as discussed. The distance from the pinhole to the detector is fixed and the pinhole aperture determines the neutron flux, 


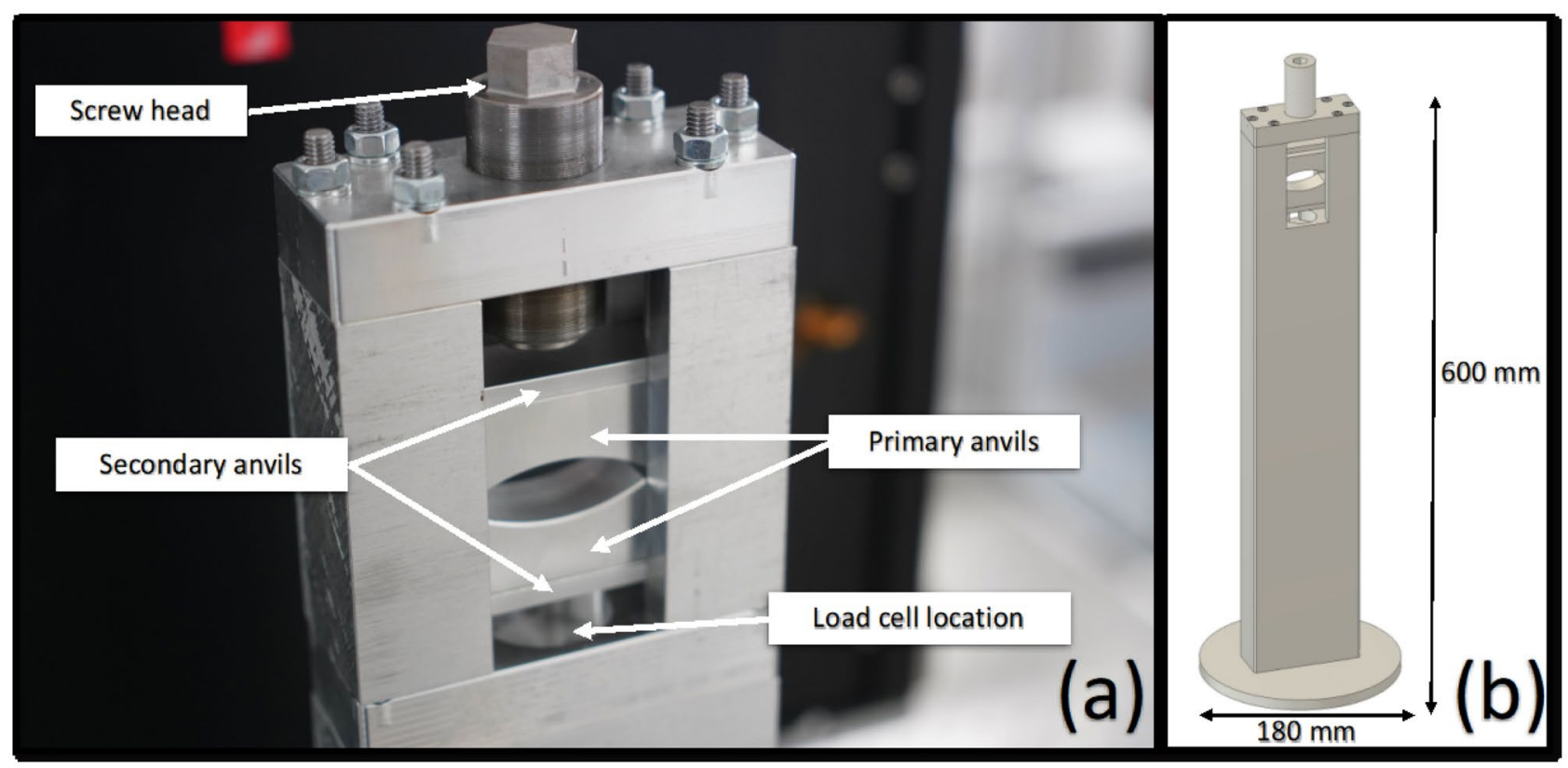

Fig. 2 Neutron imaging loading rig: (a) close up view of anvils and (b) 3D CAD (computer-aided design) model

which affects the acquisition time for sufficient signal (i.e., neutron count). This means that the main way to reach a suitable resolution for image analysis whilst maintaining a high neutron flux is to reduce the sample to active area distance and bring the sample as close to the detector as possible. Preliminary acquisitions are necessary to determine the most appropriate configuration (i.e., pinhole size, collection time, sample-to-detector distance, and chopper speed).

A proof-of-concept loading apparatus was designed to suit the requirements of close proximity to the detector, low neutron absorption, limited image shadowing and controlled loading capabilities. The rig is a manually-loaded screw-driven device for the application of compressive stress (Fig. 2). In this work, diametral compression was applied for comparison with other studies of graphite [24, 25] though other samples suited for compressive loading can be studied using the same rig.

The overall thickness of the rig was minimised to allow a short sample to active-area distance. The top assembly, or loading head, can be positioned in the neutron beam whilst avoiding potential contact with the MCP detector, which is introduced via a robotic arm. Figure 2 presents the top assembly with its main components. A loading cell $(20 \mathrm{kN})$ is located on the lower top assembly and is connected via universal serial bus to a computer for direct monitoring of the applied load. All the structural components, other than stainless steel fixing screws and bolts that are not in the beam path, are made of aluminium alloy (HE30-6082T6) for neutron transparency and sufficient mechanical strength. The curved surface of the secondary anvils is suitable for discs with a diameter between 15 and $25 \mathrm{~mm}$, maintaining the contact surface within the range specified by ASTM D8289-19 [37]. In the main configuration, the flat surface of the secondary anvils is loaded by the primary anvils. Both anvil pairs slide on two cylindrical pillars attached to the top and bottom parts of the assembly. The secondary anvils can be inverted to offer a flat loading surface for flattened discs [38] or other flat-ended compression samples.

\section{Experimental Setup}

Three days of beamtime were allocated on IMAT under experiment number RB1910517 [20]. The neutron wavelength at which the graphite basal planes will enter the (002) Bragg condition is significantly away from the $2.6 \AA$ pulse maximum at $6.7 \AA$, so for this proof-of concept experiment we have selected a pinhole aperture of $40 \mathrm{~mm}$ to collect radiographs with a resolution sufficient to identify extinction spots and compensated the loss in flux with an acquisition time of $120 \mu \mathrm{A}$ to ensure sufficient neutron count in the energy band of interest. We have also configured the disk choppers for a $10 \mathrm{~Hz}$ frequency and a phase delay of $20 \mathrm{~ms}$ moving the Time-of-Flight (ToF) range to 30-130 ms which is equivalent to approximately $2-10 \AA$; the suitability of this range is illustrated by the neutron count in Fig. 1. The data is binned and saved as a stack of images in the FITS format (Flexible Image Transport System), each image representing an approximate energy. In this experiment we have divided the data collected in 2,474 bins. 

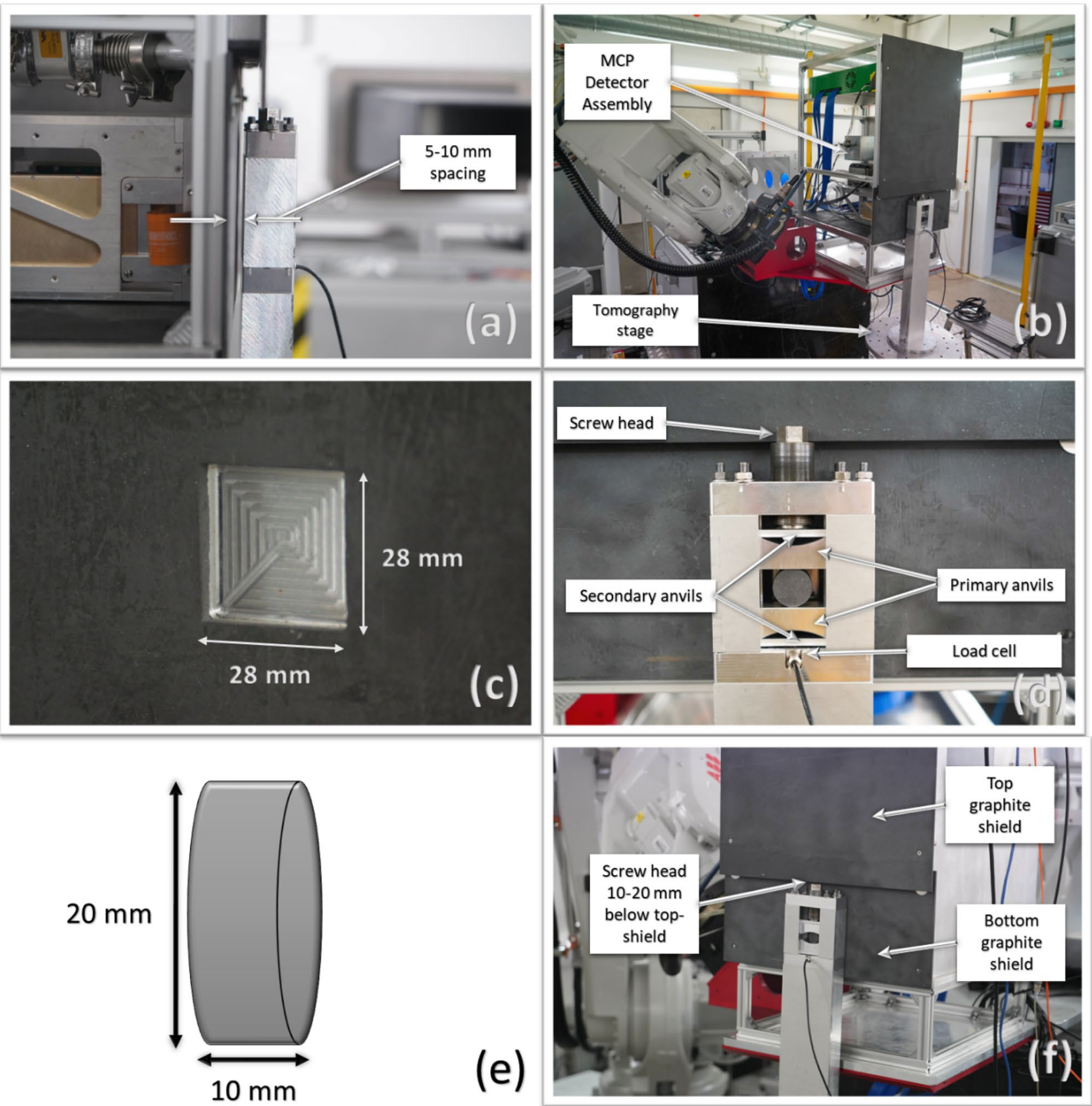

Fig. 3 Experimental setup depicting a side view with rig-to-shield distance (a), overall view with robot-arm and rig fixed on tomography stage (b), detector window close-up view (c), secondary setup close-up with screw head away from top-shield (d), graphite sample dimensions (e), and primary setup with screw head under top shield (f)

Figure 3 presents the experimental setup inside the hutch. In order to avoid the risk of collision with the detector, we have maintained a minimum of $5 \mathrm{~mm}$ distance between the rig and the lower reflector shield, and for this experiment we set the default distance to be $9 \mathrm{~mm}$. The distance from the rig surface to the centreline of the sample being $20 \mathrm{~mm}$, and the active area of the detector being $17 \mathrm{~mm}$ behind the shield, the total detector to sample centreline distance is $46 \mathrm{~mm}$ giving a resolution of approximately $184 \mu \mathrm{m}$. The detector is held by a robotic arm at a fixed position defined by the beamline arrangements, and at a fixed distance of almost $56.4 \mathrm{~m}$ from the moderator. The rig sits on the tomography stage that can be used for vertical rotation, which is attached to the heavy-duty sample stage used for all translations.

It was important to ensure there was no other reason for the apparition of new Bragg spots in the radiography, 
other than local grain reorientation or deformation. In particular, crystals could enter or leave the Bragg condition if the sample rotates sufficiently. To check for outof-plane movements and sample rotations with applied load, we used Digital Image Correlation (DIC) to monitor control experiments that were performed in the laboratory using the same loading apparatus and similar experimental conditions. DIC is a strain measurement technique developed in the 1980s based on pattern-matching of time-series greyscale images [39-41]. Subsets containing tens of pixels of an image are compared to a reference image by homogeneous linear mapping of the intensity pattern. This allows tracking of relative displacements that are smaller than a pixel and mapping of the displacement field, from which the strain field can be derived. Stereo-DIC, using two cameras, is able to provide inplane and out-of-plane displacements within the range specified by the measured volume and the depth of field. In particular, the GOM Correlate algorithm used in this study uses the principle of 3D mesh correlation and parametric inspection. The grey value distribution in each facet of the right and left camera images are reidentified to a reference set of images and 3D coordinates are triangulated [42]. A cube containing patterns with known distances is used to calibrate the stereo-DIC system. Due to the IMAT beamline configuration, it is currently not possible to use stereo-DIC during a neutron experiment, but this would be advantageous in future studies.

We employed stereo-DIC in the laboratory tests, using a $2448 \times 2050$ pixels GOM Aramis 5 M setup with $75 \mathrm{~mm}$ focal lenses and the same loading conditions as in the beamline experiment. Figure 4(a) shows the image correlation setup, with the test specimen coated with a speckle pattern using an airbrush with a $0.3 \mathrm{~mm}$ nozzle. The measuring area was $15 \times 13 \mathrm{~mm}$ with a calibration deviation of 0.028 pixels and a depth of field of $5.4 \mathrm{~mm}$ (Fig. 4(b)). The displacement field was along the beam axis, by DIC analysis with a 32-pixel facet size and a point distance of 19 pixels. The out-of-plane displacements, for an applied load of $5.2 \mathrm{kN}$ (close to failure of the specimen) are presented as an example (Fig. 4(c)) that also shows the local effects of the contact load. The average 3D tilts of the specimen surface (Fig. 4(d)) show there is a maximum rotation of approximately $0.1^{\circ}$ about the vertical axis, and a forward tilt of less than $0.2^{\circ}$ even at high load as failure is approached. The supplementary material details the digital radiography correlation used to validate the in-situ loading regime. The angular width of the Bragg edge is approximately $3.9^{\circ}$, and we can conclude from the DIC analysis that such sample rotations during the experiment would have a marginal effect.

\section{Data Processing Methodology}

A program has been developed in MATLAB for processing the radiographs and identifying local changes in transmitted intensity, available free to use under the GNU General Public License v3.0 [43]. The code is compiled as a stand-alone executable file offering a Graphical User Interface (GUI), and the source code is available from the corresponding author on request. It was developed because specific operations need to be performed on the radiographs at pixel level, so it was not possible to rely on existing codes. The code works directly with the raw datasets and is optimised for multi-thread processing; GPU acceleration is under development. It has been written to minimise the use of randomaccess memory at the expense of more frequent access to the external storage. This makes the code compatible with computers with limited specifications while having the flexibility of using the computing power of high-end workstations.

Scaling and overlap correction are applied to the raw datasets produced by the MCP detector in the FITS format. Overlap occurs due to neutrons arriving after the end of a shutter insertion and this is corrected using Poisson's statistics. Scaling of the open beam datasets is preferable for a more accurate flat-field correction as all datasets may not have the same number of triggers. The methods used for overlap and scaling correction are the same as those already used on IMAT, for which a detailed description is available in [44]. This initial pre-processing stage is applied to all radiographs, which are thereafter saved as unsigned 16-bit TIFF images.

The paths to the working folder and stages of the data acquisition in the experiment are defined, then the datasets are listed and checked for consistency. Any subsequent image pre-processing is carried out in an iterative scheme, for each image and repeated as many times as required, and only the final outputs are saved in random-access memory. Radiographs are first converted to double floating-point precision with no range limits to avoid truncation or saturation. The code initially works at a pixel level to reduce noise and to remove and replace dead or abnormal pixels. This is achieved by linear interpolation of pixels falling outside the $1-100$ percentile threshold to remove dead pixels. A shape-preserving piecewise cubic spline interpolation, of pixels with values more than three local scaled median absolute deviations from the local median within a $3 \times 3$ pixels window, replaces abnormal pixels. Pixels that suffer from detector edge effects at the border of the radiograph are removed by an initial crop of 6 pixels at each border that is maintained throughout the experiment. Flat-field correction is applied to radiographs individually and iteratively to remove detector aberrations, especially the missing lines between the 4 
(a)
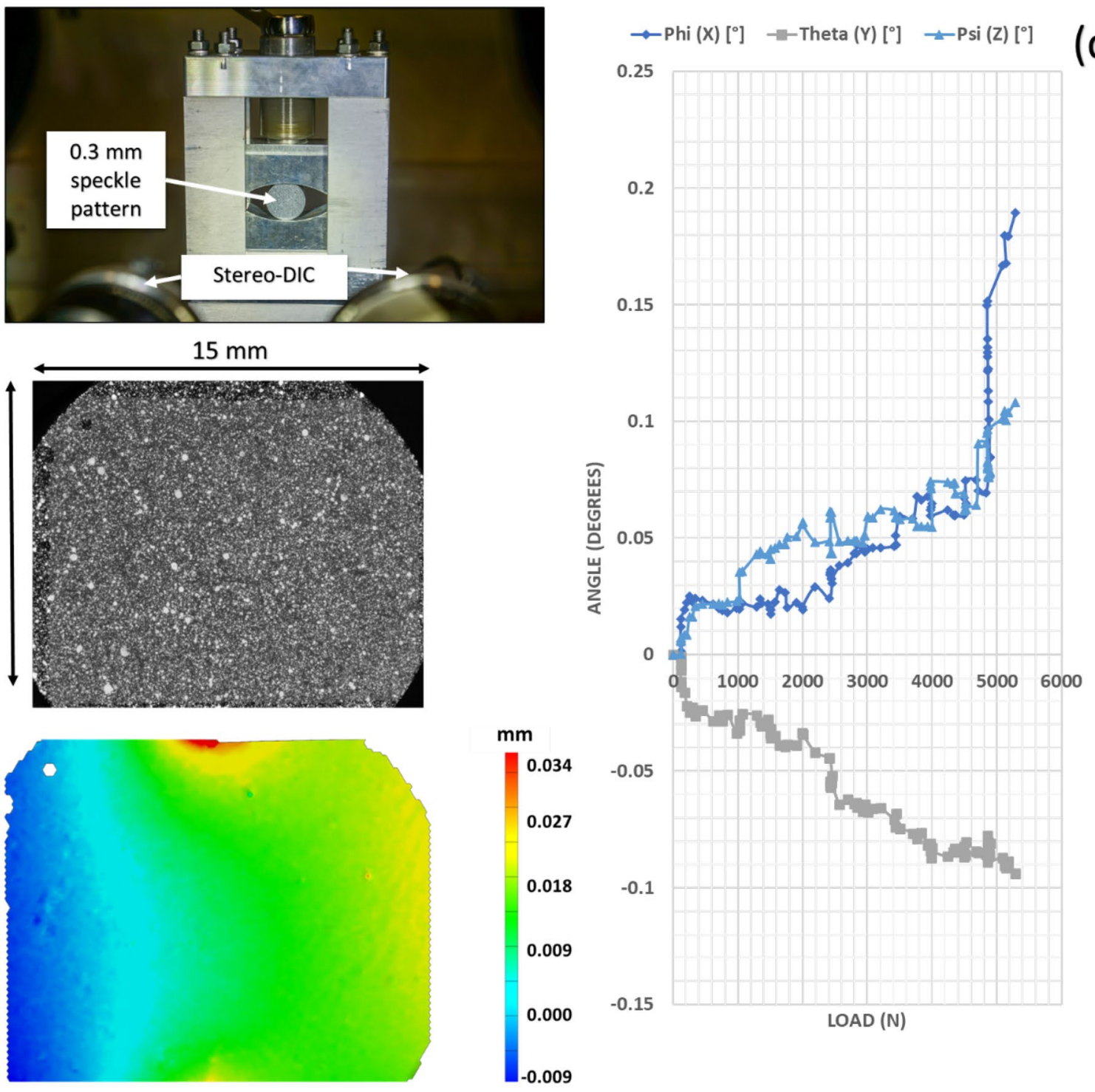

(b)

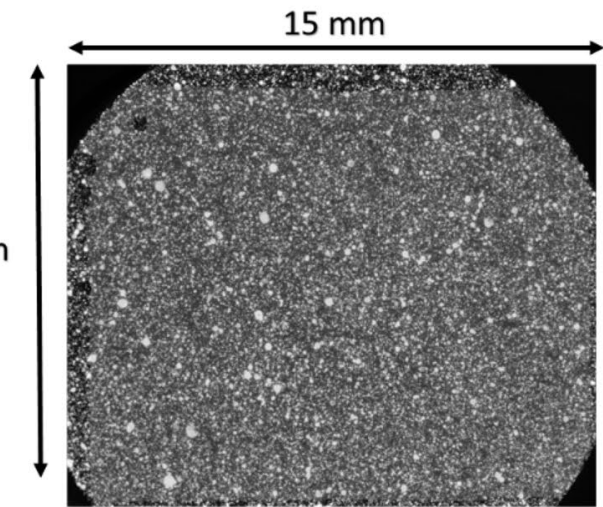

\section{(c)}
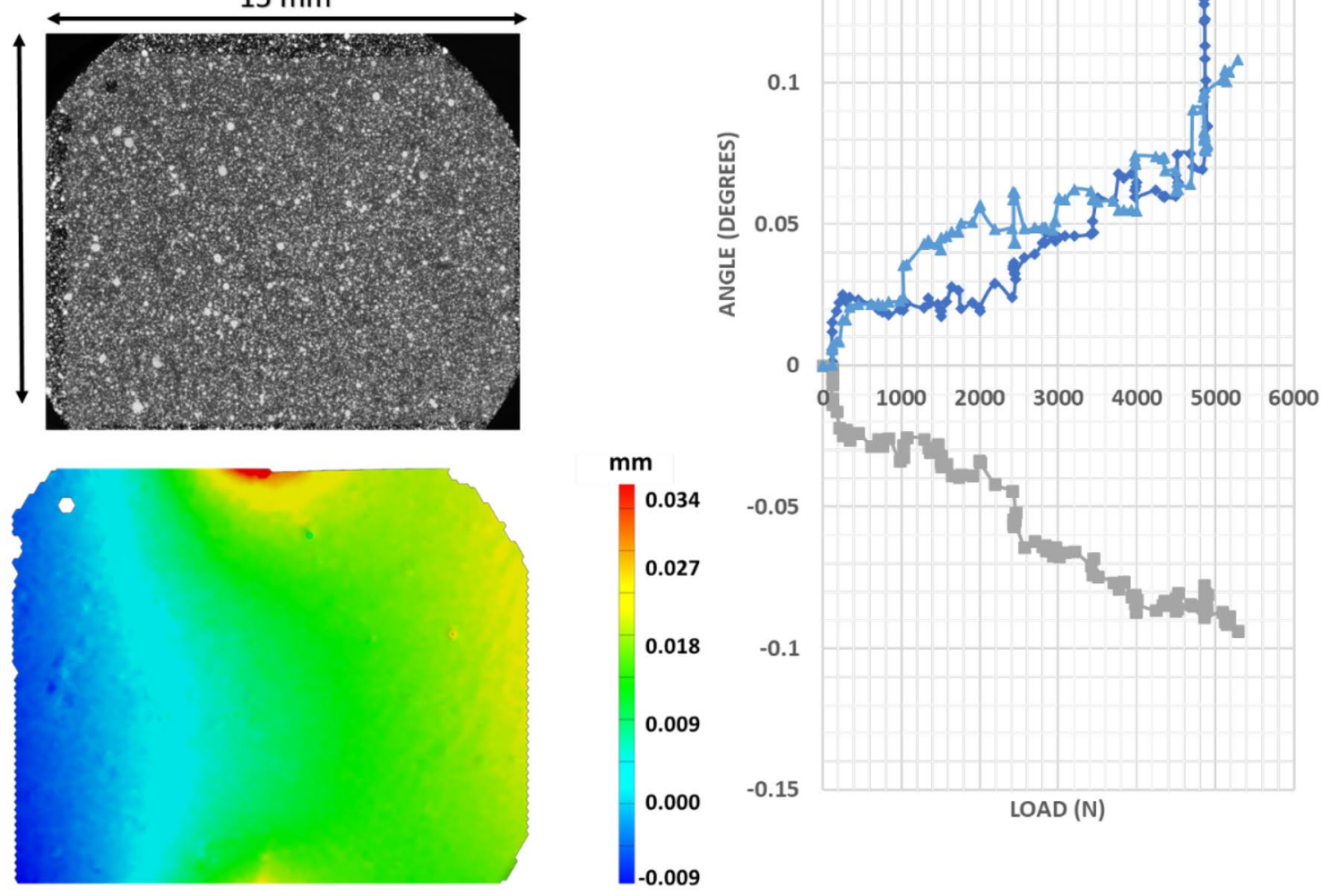

Fig. 4 (a) Stereo digital image correlation setup; (b) mapping area with speckle pattern; (c) out-of-plane z-displacements of the same area; and (d) angular rotations with load of the mapped area about the $\mathrm{X}, \mathrm{Y}$ and $\mathrm{Z}$ axes

ASIC chips of the Timepix sensor and other dust particles, and to obtain the transmitted image sequence using equation (3):

$T_{n}=\frac{R_{n}}{O B_{n}} \times f_{O B_{n}}$

where $f_{O B n}$ is the mean value of the Open Beam radiography $O B n$ representing neutrons with time of flight $n$, which divides the stage radiography image $R_{n}$ to obtain the transmitted image $T_{n}$ with neutrons with time-of-flight $\mathrm{n}$. The sub-program that is tasked with obtaining the transmission spectrum and automatically selecting the wavelength ranges uses a scaling factor $f_{O B_{n}}$ of 100 to obtain the percentage neutron transmission in the sample.
A subtraction method is used to detect changes in the transmitted image between loading stages. This requires each image to be registered with a reference in terms of deformation and movement. To address the effects of deformation of the specimen (i.e., compression), it is first necessary to measure the relative displacements. This could not be done using image correlation of optical images (as in Fig. 4) due to the configuration of the IMAT instrument, and so was carried out using the attenuation contrast of the radiographs. The displacement fields are obtained by DIC of averaged images in a wavelength range where it is expected there would be limited Bragg scattering. Figure 5 presents the specific case for transmission in Gilsocarbon graphite. The graphite Bragg-edge, which 

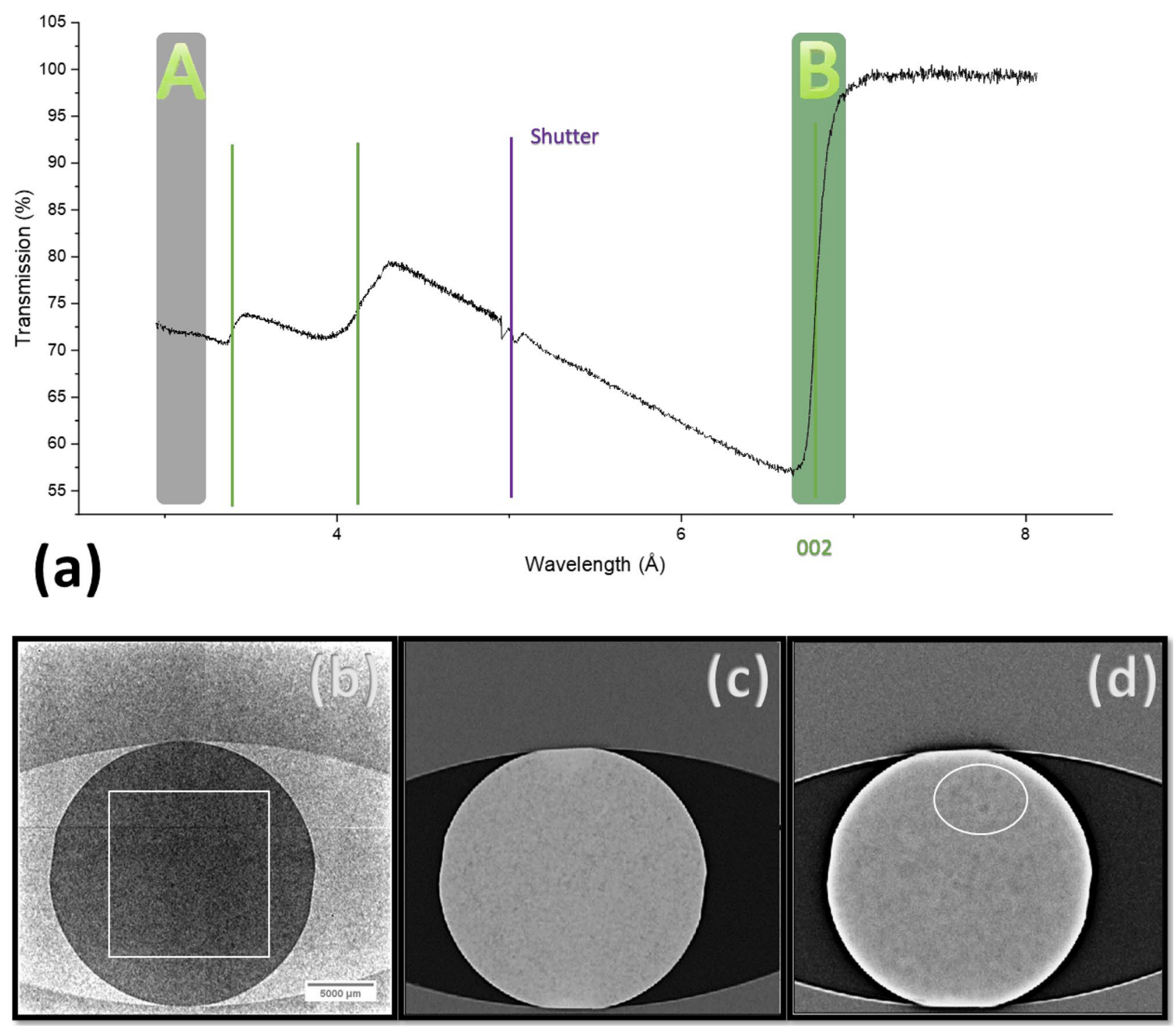

Fig. 5 (a) Regions A and B are indicated on a neutron transmission spectrum averaged over the square area that is marked in (b) the raw uncorrected radiography at $2.9 \mathrm{~A}$; (c) pre-processed and averaged image from Region A; and (d) from region B at the (002) Bragg-edge. [A blade with neutron absorption properties is inserted into the beam to avoid sample and detector activation when no data are collected. Even with correction for its insertion, there are residual "dips" on the transmission graph due to this 'shutter', as indicated in (a)]

corresponds to the basal plane (002) reflection, is located in region B. In region A, neutrons have a low scattering probability, but the Gilsocarbon graphite has a very heterogeneous distribution of porosity [25], and all features should be from attenuation differences in the neutron path due to absorption (e.g. density variation). At the resolution of neutron imaging this heterogeneity can be assumed to not change with load, and so it provides the 'speckle' for DIC of the specimen's deformation. Region A is centred around a user defined wavelength in a region away from any Bragg peak. Radiographs are pre-processed and then averaged over an increasing range until the signal quality is sufficient for image registration. A Perception-based
Image Quality Evaluator (PIQE) (non-referenced and unsupervised algorithm) is used at each iteration to compute a quality score. When the value of the score falls below $5 \%$ of its preceding iteration, the averaging and range extension ends.

After the averaged images are obtained, further preprocessing and image masking was done. This includes outlier removal with a shape-preserving piecewise cubic spline interpolation where there is a three-time local scaled median absolute deviation from the local median over a window length of $3 \times 3$ pixels, gaussian smoothing with a standard deviation of 0.5 , and a threshold-based segmentation to remove the loading anvils and air. To obtain images 
only of the sample, the grayscale intensity is adjusted by scaling throughout the steps to maintain a constant mean in a $50 \times 50$ pixels zone with neutron transmission through air and an algorithmic threshold segmentation is then used to mask the non-sample areas. This uses as input a mean intensity that assumes that the sample is centred (an alternative centre can be defined) and is used to inform a geometric mask for the subsequent analysis of the radiographs. This set of images, each representing an absorption contrast image of the sample at each step of the loading, is then fed to the DIC algorithm. The method uses the demons algorithm developed by Thirion [45] adapted to provide non-diffeomorphic transformations [46]. It employs a 1.5 diffusion-like gaussian regularization at six resolution levels with up to 100 iterations, to obtain the relative displacement of each pixel. The output is a regular array of displacements with an array size determined by resolution of the correlated images.

Region B, or Near Bragg-Edge (NBE), is defined around the Bragg-edge of interest. Each radiograph over this range and for stages under mechanical stress is loaded together with its corresponding open beam and reference radiographs. After image pre-processing and correction, the radiographs for each stage are registered with the displacement fields calculated using the A-region by inverse mapping. Pixel values in the result image are obtained after mapping locations to the corresponding locations in the original image by applying the inverse transformation. Nearest interpolation is applied in the input space to compute the pixel value in the registered image. The reference radiograph undergoes the same pre-processing and intensity adjustment before being compared to the registered stage radiograph by readjusted differentiation. Sequential linear combination of radiographs over the B-range subsequently reduces noise and increases the contrast of the features that are in the Bragg condition. In this analysis, the B-range represents an interval of 80 radiographs. The code allows one to split $\mathrm{B}$ into multiple subsets of radiographs.

\section{Preliminary Results}

\section{Data Quality}

The constraints related to the sample-to-detector distance and the pinhole for this preliminary experiment meant that the signal to noise ratio of the radiography was quite low, especially away from the instrument's maximum energy peak. In any configuration it is necessary to work over a range of energies for image processing to be feasible, however low signal meant that the range had to be quite wide in this experiment. Figure 5 presents a single raw and uncorrected radiograph in the A-range for the $0^{\text {th }}$ pre-load stage
$(0.2 \mathrm{kN})$ together with a corrected and averaged image over the A-range and over the B-range. The latter clearly presents contrast from features that are only visible at the Bragg-edge characteristic, with those crystals in the Bragg condition forming a dark extinction spot. The contrast of these features is relatively low within a single radiograph. The width of the Bragg edge [21] and the range of energy selected for summation will affect the visibility of these features in the processed images.

As seen in Fig. 5 (b), the raw radiograph has a weak signal. It represents all the neutrons that arrived on the detector with a wavelength of $2.9 \AA$ over an acquisition time of three hours. The energy step between radiographs is approximately $0.0014 \AA$ until $4.96 \AA$ and $0.0028 \AA$ to the maximum at $8.06 \AA$. This raw image also reveals the four plates forming the detector as well as the dead or invalid pixels that need to be removed. As radiographs were obtained in the highest flux region of the instrument's spectrum (Fig. 1) and away from the Bragg edge of interest, it was only necessary to work over a small range of energy to perform the image correlation for measurement of sample deformation, from $2.9 \AA$ to $3.23 \AA$.

During the data acquisition, the load was allowed to settle after each step before collecting the signal from the neutrons. Further stress relaxation led to a small difference of about $50 \mathrm{~N}$ over the acquisition time of approximately $3 \mathrm{~h}$. Since radiographs do not represent a successive exposure acquisition but rather a collection of neutrons at different energies, we can consider that all radiographs are acquired at the same time.

\section{Image Registration}

Realignment of energy-resolved images has not been attempted before. Displacements fields were obtained from pre-processed, corrected, averaged and masked images over the A-range. The relative vertical and horizontal fields for the $5^{\text {th }}$ stage $(4 \mathrm{kN})$ compared to the reference (0th pre-load stage at $0.2 \mathrm{kN}$ ) are presented in Fig. 6. This shows the expected bi-axial deformation and compares well to previous correlation studies performed on similar specimens [28]. These particular sets of images have also been correlated using commercial DIC software (LaVision DaVis 8.40) and a comparison that verifies the performance of the demon code is presented in the supplementary materials. Figure 6(a) and (b) show the displacement fields that registered the images. The coloured regions in Fig. 6(c) and (d) represent areas of image intensity mismatch, where the disc (and features in its microstructure) and the anvil do not overlap. The realigned and deformed image is shown to correctly fit (i.e. register with) the reference in Fig. 6(d). Since radiographs inside the A-range (away from the Bragg edge) have the same level of deformation as those in the 

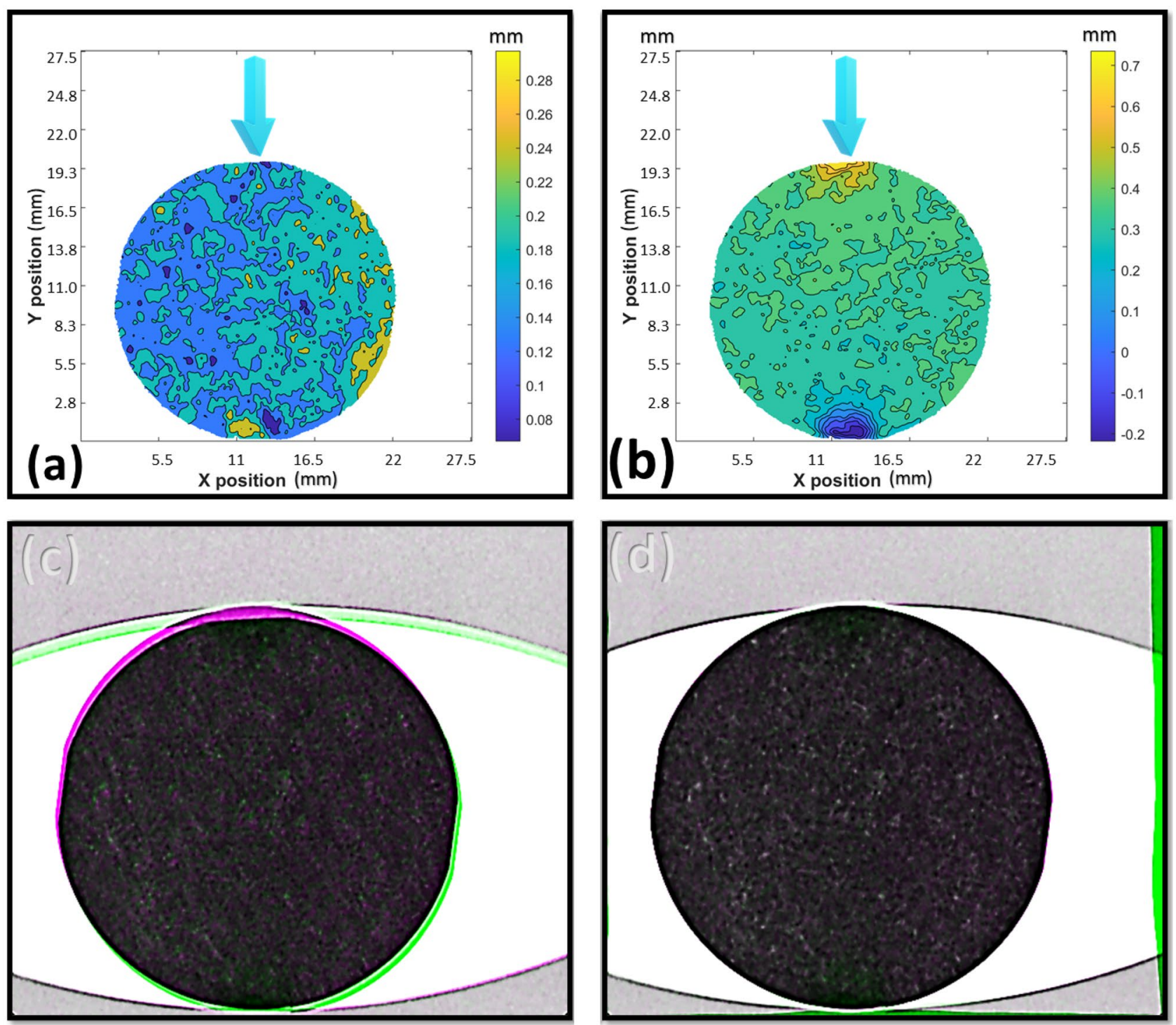

Fig. 6 Comparison of 5 th stage $(4 \mathrm{kN})$ to reference ( 0 th pre-load stage at $0.2 \mathrm{kN})$ : (a) horizontal displacements; (b) vertical displacements; (c) unregistered image (averaged for energies over the A-range) and (d) registered image

B-range, the measured displacement fields can be used to register the microstructure images in the B-range, in which extinction spots are observed that will be discussed in detail in Sect. 3.3.

\section{Observations}

The final averaged images after differentiation processing of Bragg edge radiographs in region B are presented in Fig. 7. A region near the specimen centre (identified by the dashed square) is presented as a close-up view in Fig. 8. Within this, several features are highlighted in circles. The images show there are some features at $1 \mathrm{kN}$ that persist in all subsequent steps (albeit with some change in shape and contrast) of the loading sequence. We deduce the dark persistent features are extinction spots that are not present in the reference stage. Since the attenuation contrast does not change, they are distinct also from the microstructure attenuation. Similarly, bright spots would arise from grains that were in extinction in the reference image and are no longer in this condition. This points to local changes in scattering due to grain reorientation or plastic deformation [17, 47-49].

The exact number or spatial distribution of these features are yet to be determined. This would allow correlations between the applied strain and grain deformation in Gilsocarbon graphite to be examined. Nonetheless, comparison of Fig. 8(c), (d) and (e) suggests that the local change in Bragg contrast might be recovered upon unloading. The feature highlighted by the dashed circle in Fig. 8 is mostly visible at the highest load step of $4 \mathrm{kN}$ and appears to return to a 
Fig. 7 Processed radiographs from energy region $B$, with increasing load in observation steps from (a) to (d) $(1 \mathrm{kN}$, $2 \mathrm{kN}, 3 \mathrm{kN}, 4 \mathrm{kN}$, with respect to stage 0 ) and then (e) unloading to $2 \mathrm{kN}$. The dashed square in (a) identifies the feature of interest presented in Fig. 8
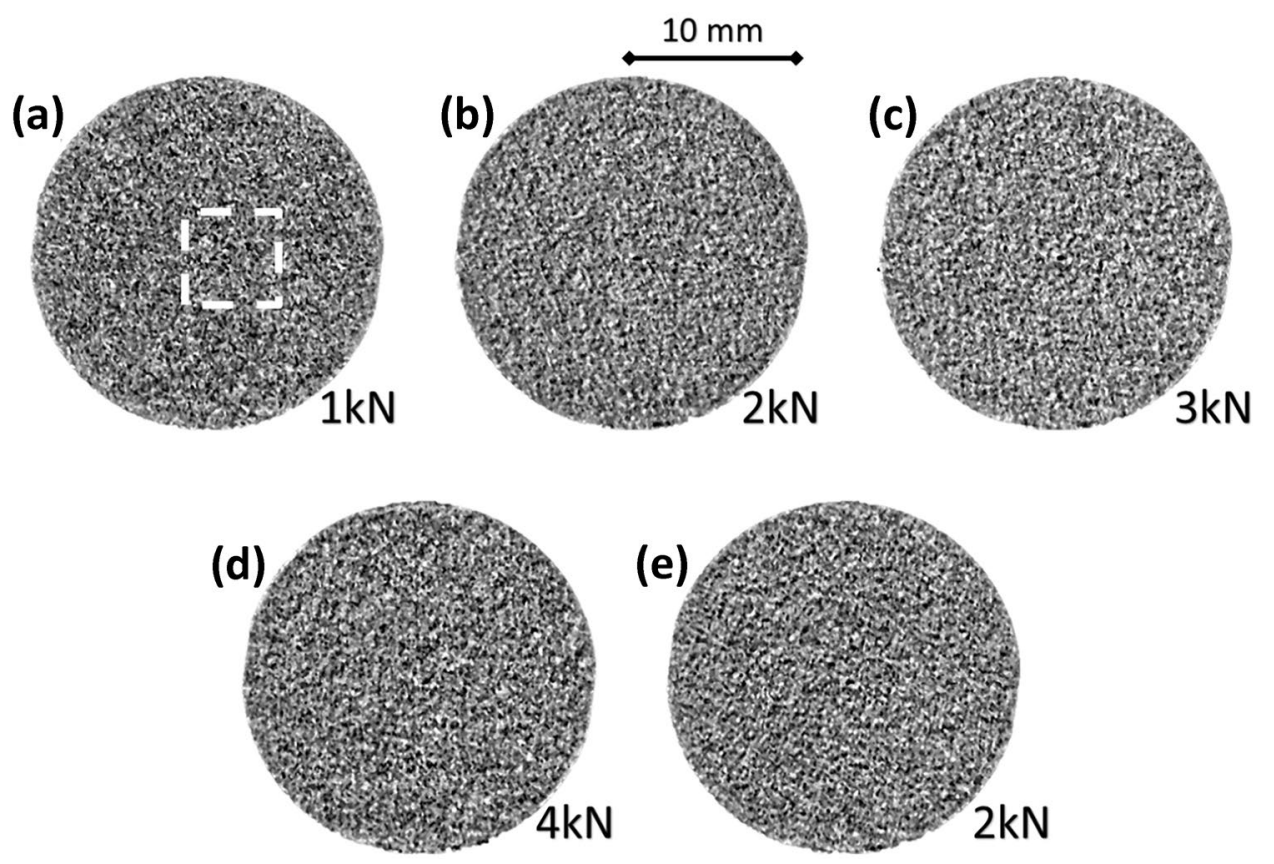

similar appearance as that of the $2 \mathrm{kN}$ step when unloaded. The other feature (solid circle in Fig. 8) persists without any significant change throughout the sequence.

To quantify the general persistence of the contrast features, Fig. 9 presents the results of an image correlation analysis applied to the resultant images of stages 2 to 5 , with respect to stage $1(1 \mathrm{kN})$ (i.e. the images in Fig. 7). The analysis was done using the least means squares algorithm with a moving window of 21 pixels and a step size of 4 pixels in the LaVision DaVis StrainMaster 8.4 software, with the objective of mapping the degree of correlation. Image noise and changes in Bragg contrast from grain orientation would lead to low correlation, whereas persistent patterns of features will have higher correlation. The colour scale in Fig. 9 represents the correlation value, such that the zones with correlation value exceeding 0.5 have a high match probability with respect to stage 1 . The generally high values distributed across the image affirm that the contrast features in the processed difference radiographs are dominated by signal from Bragg diffraction in the microstructure, rather than noise. We can observe that some regions maintain higher correlation throughout, whereas others change in intensity
Fig. 8 Close-up view at the centre of the specimen $(90 \times 80$ pixels) (region indicated in Fig. 7(a) (a)

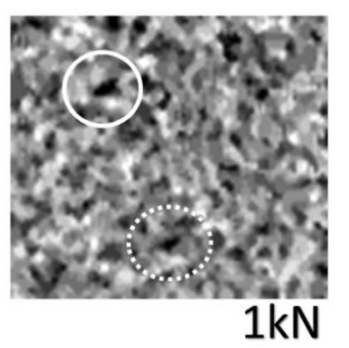

(b)

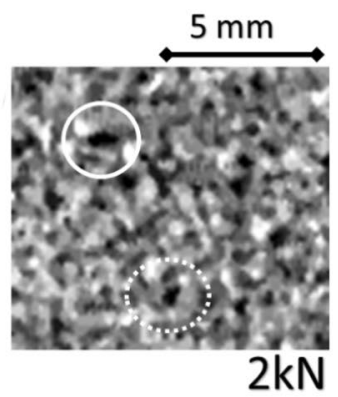

(c)

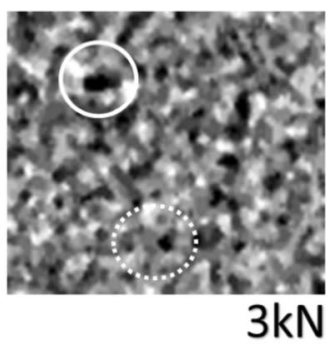

(d)

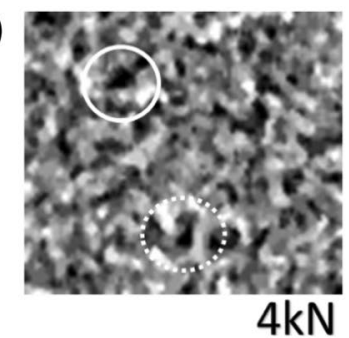

(e)

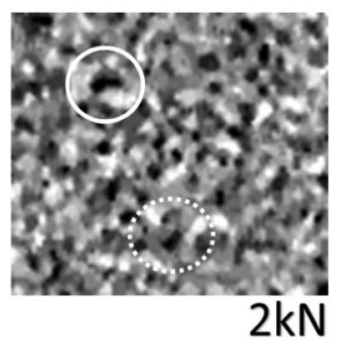




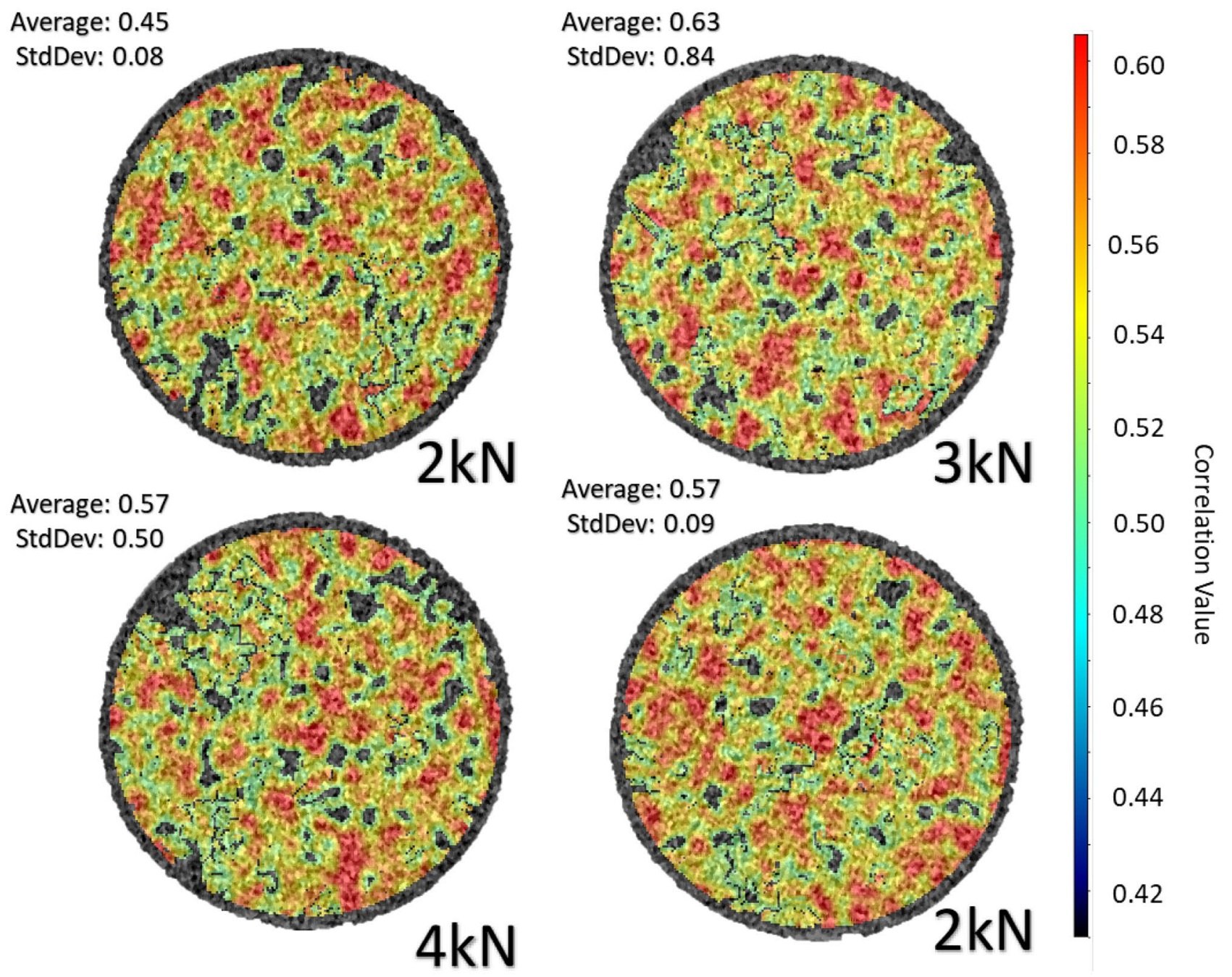

Fig. 9 Correlation values for the comparison to stage 1 difference image at $1 \mathrm{kN}$, zero values are not counted in the standard deviation or the mean

or shape or appear and disappear. It is interesting to note that correlation with respect to stage 1 appears generally higher for both images at $2 \mathrm{kN}$ (i.e., stage 2 , loading and stage 5 , unloading). A more detailed analysis of the persistence of high correlation regions, and local decreases in correlation that would indicate changes in Bragg contrast, is feasible but this will require raw data with higher signal to noise for higher confidence.

\section{Discussion}

The loading rig has been designed for brittle and quasibrittle materials, and the Brazilian disc mechanical testing method, whose use for graphite is supported by a relevant standard [37], is routinely applied to other such materials including cements and geological materials. The developed method offers a means to investigate crystal reorientation or deformation for materials whose grain size, mosaicity and/or surface roughness would not make it practical for any other more straightforward studies. On IMAT, in the best-case scenario, where the $20 \mathrm{~mm}$ aperture is sufficient to provide data within a reasonable experimental timeframe, the best achievable resolution would be $70 \mu \mathrm{m}$, which is just above the ASIC sensor pixel size of $55 \mu \mathrm{m}$. This method is most suited to investigate grain reorientation and deformation in large grain samples, with potential applications in materials such as uranium [50], or those with long range domain structures such as titanium alloys [51, 52].

Although this technique is geared towards large samples, there is an upper limit which is simply defined by the active area of the available detector. The $28 \times 28 \mathrm{~mm}^{2}$ active detector used in this preliminary experiment constrains the 
possible sample cross-section to below $26 \times 26 \mathrm{~mm}^{2}$. A possible workaround for large samples, apart from stacking up Timepix chips in a new detector, would be to perform acquisitions at multiple regions of interest by movement of the loading rig. The resulting stacks of overlapping radiographs can then be stitched using image correlation methods; however, this would increase the experimental time significantly.

The manual loading of the rig has limitations on the maximum load that can be attained and requires user interventions at each load step. The current apparatus could be upgraded with an automatic loading system, and the next design iteration would apply loading from the bottom with a motor. This would allow for fully automatic and remotely supervised acquisitions as well as more precise load increments, and the direct tracking of displacement.

The qualitative results presented here have shown the presence of persistent and non-persistent local features from coherent Bragg scattering during loading that are different from observable microscopic features. As the imaging method is in two dimensions, the data acquired is averaged along the thickness of the sample, which may imply that some extinction spots overlap. The observed changes in the Bragg contrast might be attributed to the processes of grain reorientation or plastic deformation in the graphite crystals. A second iteration of the analysis program is under development. It includes direct segmentation of the extinction spots, subset tracking with angular dependence and quantification of the changes in image contrast. A subsequent experiment focusing specifically on Gilsocarbon graphite has recently been performed on IMAT and employed a longer count time and/or varying pinhole apertures [20]. The resulting radiographs offer a higher signal to noise ratio, which should allow specific conclusions for the case of graphite on the relation between strain and local changes in Bragg contrast that may occur from crystal reorientation by twinning or kink band formation or broadening of the Bragg edge due to plastic deformation. Further experiments using this methodology may then be conducted on irradiated samples and/or at high temperatures, to better understand how these affect the structural integrity of graphites.

\section{Conclusions}

A new technique has been presented that allows the investigation of crystal reorientation under load, by using advanced image analysis of time-of-flight energy-resolved neutron imaging. This has involved the design and manufacture of a loading device specifically adapted to the requirements of Bragg-edge imaging and the development of image analysis tools to account for sample deformation in the registration of radiographs. This bring the ability to identify Bragg extinction spots, which may then be observed to examine for reorientation.

Supplementary Information The online version contains supplementary material available at https://doi.org/10.1007/s11340-021-00754-1.

Acknowledgements The authors would like to thank Abdalrhaman Koko and Marcus Williamson (University of Oxford) for their participation in the proof-of-concept experiment RB1910517. Thomas Zillhardt is grateful for support from EDF Energy Generation UK.

Author Contributions Zillhardt: Conceptualisation, Investigation, Methodology, Software, Writing - original draft preparation. Burca: Investigation, Writing - review and editing. Marrow: Conceptualisation, Writing - review and editing. Liu: Writing - review and editing.

Funding T. Zillhardt has received research funding from EDF Energy Generation UK. The beamtime at the ISIS-IMAT instrument and associated costs have been provided by STFC after a peer-reviewed process. Genoveva Burca is an instrument scientist at the STFC Spallation Source.

\section{Declarations}

Conflict of Interest The authors have no affiliation with any company nor have any financial interest in the material presented in this manuscript.

Open Access This article is licensed under a Creative Commons Attribution 4.0 International License, which permits use, sharing, adaptation, distribution and reproduction in any medium or format, as long as you give appropriate credit to the original author(s) and the source, provide a link to the Creative Commons licence, and indicate if changes were made. The images or other third party material in this article are included in the article's Creative Commons licence, unless indicated otherwise in a credit line to the material. If material is not included in the article's Creative Commons licence and your intended use is not permitted by statutory regulation or exceeds the permitted use, you will need to obtain permission directly from the copyright holder. To view a copy of this licence, visit http://creativecommons.org/licenses/by/4.0/.

\section{References}

1. Burca G, Kockelmann W, James JA, Fitzpatrick ME (2013) Modelling of an imaging beamline at the ISIS pulsed neutron source. J Instrum 8:P10001-P10001. https://doi.org/10.1088/1748-0221/8/ 10/p10001

2. Thomason JWG (2019) The ISIS Spallation Neutron and Muon Source-The first thirty-three years. Nucl Instruments Methods Phys Res Sect A Accel Spectrometers, Detect Assoc Equip 917:61-67. https://doi.org/10.1016/j.nima.2018.11.129

3. Broomby R (2014) New cracks in Hunterston reactor - BBC News. In: BBC News Online. http://www.bbc.co.uk/news/science-environment29481481. Accessed 4 Dec 2017

4. Marsden BJ, Hall GN (2012) Graphite in Gas-Cooled Reactors. In: Comprehensive Nuclear Materials. Elsevier, pp 325-390

5. Abram T, Ion S (2008) Generation-IV nuclear power: A review of the state of the science. Energy Policy 36:4323-4330. https:// doi.org/10.1016/j.enpol.2008.09.059 
6. Kockelmann W, Minniti T, Pooley D et al (2018) Time-of-Flight Neutron Imaging on IMAT@ISIS: A New User Facility for Materials Science. J Imaging 4:47. https://doi.org/10.3390/jimaging4030047

7. Strobl M, Woracek R, Kardjilov N et al (2012) Time-of-flight neutron imaging for spatially resolved strain investigations based on Bragg edge transmission at a reactor source. Nucl Instruments Methods Phys Res Sect A Accel Spectrometers, Detect Assoc Equip 680:27-34. https://doi.org/10.1016/j.nima.2012.04.026

8. Strobl M, Hilger A, Boin M, et al (2011) Time-of-flight neutron imaging at a continuous source: Proof of principle using a scintillator CCD imaging detector. In: Nuclear Instruments and Methods in Physics Research, Section A: Accelerators, Spectrometers, Detectors and Associated Equipment. North-Holland, pp 149-155

9. Woracek R, Penumadu D, Kardjilov N et al (2011) Neutron Braggedge-imaging for strain mapping under in situ tensile loading. J Appl Phys 109:93506. https://doi.org/10.1063/1.3582138

10. Strickland J, Tassenberg K, Sheppard G et al (2020) 2D single crystal Bragg-dip mapping by time-of-flight energy-resolved neutron imaging on IMAT@ISIS. Sci Rep 10:20751. https://doi.org/ 10.1038/s41598-020-77572-3

11. Song G, Lin J, Bilheux J et al (2017) Characterization of Crystallographic Structures Using Bragg-Edge Neutron Imaging at the Spallation Neutron Source. J Imaging 3:65. https://doi.org/10. 3390/jimaging3040065

12. Tremsin AS, Vallerga JV, McPhate JB et al (2013) High Resolution Photon Counting With MCP-Timepix Quad Parallel Readout Operating at $>1 \mathrm{KHz}$ Frame Rates. IEEE Trans Nucl Sci 60:578585. https://doi.org/10.1109/TNS.2012.2223714

13. Shen K, Yu S, Kang F (2019) The microstructure and texture of Gilsocarbon graphite. Carbon N Y 153:428-437. https://doi.org/ 10.1016/j.carbon.2019.06.108

14. Reischig P, King A, Nervo L et al (2013) Advances in X-ray diffraction contrast tomography: flexibility in the setup geometry and application to multiphase materials. J Appl Crystallogr 46:297311. https://doi.org/10.1107/S0021889813002604

15. King A, Ludwig W, Herbig M et al (2011) Three-dimensional in situ observations of short fatigue crack growth in magnesium. Acta Mater 59:6761-6771. https://doi.org/10.1016/J.ACTAMAT. 2011.07.034

16. King A, Johnson G, Engelberg D, et al (2008) Observations of Intergranular Stress Corrosion Cracking in a Grain-Mapped Polycrystal. Science (80- ) 321:382 LP - 385

17. Gonzalez D, King A, Mostafavi M et al (2013) Three-dimensional observation and image-based modelling of thermal strains in polycrystalline alumina. Acta Mater 61:7521-7533. https://doi.org/10. 1016/J.ACTAMAT.2013.06.005

18. Gustafson S, Ludwig W, Shade P et al (2020) Quantifying microscale drivers for fatigue failure via coupled synchrotron X-ray characterization and simulations. Nat Commun 11:3189. https:// doi.org/10.1038/s41467-020-16894-2

19. Cereser A, Strobl M, Hall SA et al (2017) Time-of-Flight Three Dimensional Neutron Diffraction in Transmission Mode for Mapping Crystal Grain Structures. Sci Rep 7:9561. https://doi.org/10. 1038/s41598-017-09717-w

20. Marrow TJ, Zillhardt T, Williamson M et al (2020) RB1910517-1: High Resolution Neutron Bragg Imaging of Gilsocarbon Graphite for the Investigation of Crystal Reorientation @IMAT. STFC ISIS Neutron Muon Source. https://doi.org/10.5286/ISIS.E.RB1910517-1

21. Fermi E, Sturm WJ, Sachs RG (1947) The Transmission of Slow Neutrons through Microcrystalline Materials. Phys Rev 71:589_ 594. https://doi.org/10.1103/PhysRev.71.589

22. Reid A, Martinez I, Marshall M, et al (2018) Mapping of axial plastic zone for roller bearing overloads using neutron transmission imaging. Mater Des 156:103-112. https://doi.org/10.1016/j. matdes.2018.06.042
23. Marsden BJ, Hall GN, Wouters O et al (2008) Dimensional and material property changes to irradiated Gilsocarbon graphite irradiated between 650 and $750{ }^{\circ} \mathrm{C}$. J Nucl Mater 381:62-67. https:// doi.org/10.1016/j.jnucmat.2008.07.018

24. Laudone GM, Gribble CM, Matthews GP (2014) Characterisation of the porous structure of Gilsocarbon graphite using pycnometry, cyclic porosimetry and void-network modeling. Carbon $\mathrm{N}$ Y 73:61-70. https://doi.org/10.1016/j.carbon.2014.02.037

25. Marsden BJ, Haverty M, Bodel W et al (2016) Dimensional change, irradiation creep and thermal/mechanical property changes in nuclear graphite. Int Mater Rev 61:155-182. https:// doi.org/10.1080/09506608.2015.1136460

26. Mattes M, Keinert J (2005) Status of thermal neutron scattering data for graphite

27. Chung DDL (2002) Review Graphite. J Mater Sci 37:1475-1489. https://doi.org/10.1023/A:1014915307738

28. Liu D, Zillhardt T, Earp P et al (2020) In situ measurement of elastic and total strains during ambient and high temperature deformation of a polygranular graphite. Carbon N Y 163:308-323. https:// doi.org/10.1016/j.carbon.2020.03.020

29. Soule DE, Nezbeda CW (1968) Direct Basal-Plane Shear in SingleCrystal Graphite. J Appl Phys 39:5122-5139. https://doi.org/10. 1063/1.1655933

30. Barsoum MW, Murugaiah A, Kalidindi SR, et al (2004) Kink bands, nonlinear elasticity and nanoindentations in graphite. Carbon N Y 42:1435-1445. https://doi.org/10.1016/j.carbon.2003.12. 090

31. Palache C (1941) Contributions to the mineralogy of sterling hill, new jersey: morphology of graphite, arsenopyrite, pyrite, and arsenic. Am Mineral 26:709-717

32. Freise EJ, Kelly A (1961) Twinning in Graphite. Proc R Soc Lond A Math Phys Sci 264:269-276

33. Kennedy AJ (1960) Dislocations and Twinning in Graphite. Proc Phys Soc 75:607-611. https://doi.org/10.1088/0370-1328/75/4/ 314

34. Skinner J, Gane N (1973) The deformation and twinning of graphite crystals under a point load. Philos Mag A J Theor Exp Appl Phys 28:827-837. https://doi.org/10.1080/14786437308220986

35. Liu D, Cherns D, Johns S, et al (2021) A macro-scale ruck and tuck mechanism for deformation in ion-irradiated polycrystalline graphite. Carbon N Y 173:215-231. https://doi.org/10.1016/j. carbon.2020.10.086

36. Heard PJ, Wootton MR, Moskovic R, Flewitt PEJ (2011) Deformation and fracture of irradiated polygranular pile grade A reactor core graphite. J Nucl Mater 418:223-232. https://doi.org/10. 1016/j.jnucmat.2011.07.003

37. ASTM D8289 - 19 Standard Test Method for Tensile Strength Estimate by Disc Compression of Manufactured Graphite. https:// www. astm.org/DATABASE.CART/HISTORICAL/D8289-19. htm. Accessed 20 Nov 2020

38. Joyce MR, Marrow TJ, Mummery P, Marsden BJ (2008) Observation of microstructure deformation and damage in nuclear graphite. Eng Fract Mech 75:3633-3645. https://doi.org/10.1016/J. ENGFRACMECH.2007.11.003

39. Peters WH, Ranson WF (1982) DIGITAL IMAGING TECHNIQUES IN EXPERIMENTAL STRESS ANALYSIS. Opt Eng 21:427-431. https://doi.org/10.1117/12.7972925

40. Sutton MA, Wolters WJ, Peters WH, et al (1983) Determination of displacements using an improved digital correlation method. Image Vis Comput 1:133-139. https://doi.org/10.1016/02628856(83)90064-1

41. McNeill SR, Peters WH, Sutton MA (1987) Estimation of stress intensity factor by digital image correlation. Eng Fract Mech 28:101-112. https://doi.org/10.1016/0013-7944(87)90124-X

42. (2015) GOM Correlate Professional V8 SR1 Manual Basic Inspection-3D Testing 
43. Zillhardt T (2021) NEUTRONIX: Program for the detection of grain reorientation with in-situ Bragg imaging. https://github.com/ olayne/NEUTRONIX

44. Liptak A, Burca G, Kelleher J et al (2019) Developments towards Bragg edge imaging on the IMAT beamline at the ISIS Pulsed Neutron and Muon Source: BEAn software. J Phys Commun. https://doi.org/10.1088/2399-6528/ab5575

45. Thirion J-P (1998) Image matching as a diffusion process: an analogy with Maxwell's demons. Med Image Anal 2:243-260. https:// doi.org/10.1016/S1361-8415(98)80022-4

46. Vercauteren T, Pennec X, Perchant A, Ayache N (2009) Diffeomorphic demons: Efficient non-parametric image registration. Neuroimage 45:S61-S72. https://doi.org/10.1016/j.neuroimage. 2008.10.040

47. Renversade L, Quey R (2019) Analysis of in-grain orientation distributions in deformed aluminium using X-ray diffraction and finite elements. In: IOP Conference Series: Materials Science and Engineering. IOP Publishing Ltd, p 012022

48. Nakai Y, Shiozawa D, Kikuchi S, et al (2019) Observations of twinning and detwinning in magnesium alloy by synchrotron radiation DCT and EBSD. In: Procedia Structural Integrity. Elsevier B.V., pp 83-88

49. Proudhon H, Guéninchault N, Forest S, Ludwig W (2018) Incipient Bulk Polycrystal Plasticity Observed by Synchrotron In-Situ
Topotomography. Materials (Basel) 11:2018. https://doi.org/10. 3390/ma11102018

50. Earp P, Kabra S, Askew J, Marrow TJ (2018) Lattice strain and texture development in coarse-grained uranium-a neutron diffraction study. 12012. https://doi.org/10.1088/1742-6596/1106/1/ 012012

51. Hoye N, Li HJ, Cuiuri D, Paradowska A (2014) Measurement of residual stresses in titanium aerospace components formed via additive manufacturing. In: Materials Science Forum. Trans Tech Publications Ltd, pp 124-129

52. Nervo L, King A, Fitzner A et al (2016) A study of deformation twinning in a titanium alloy by X-ray diffraction contrast tomography. Acta Mater 105:417-428. https://doi.org/10.1016/j.actamat. 2015.12.032

53. Shoemake K (1994) Euler Angle Conversion. In: Heckbert PSBTGG (ed) Graphics Gems. Elsevier, pp 222-229

Publisher's Note Springer Nature remains neutral with regard to jurisdictional claims in published maps and institutional affiliations. 